\title{
Improved Determination of Surface and Atmospheric Temperatures Using Only Shortwave AIRS Channels: The AIRS Version 6 Retrieval Algorithm
}

\author{
Joel Susskind $^{1}$, John Blaisdell ${ }^{2}$, and Lena Iredell ${ }^{2}$ \\ ${ }^{1}$ NASA Goddard Space Flight Center, Greenbelt, MD, USA 20771 \\ ${ }^{2}$ SAIC, NASA Goddard Space Flight Center, Greenbelt, MD, USA 20771
}

AIRS was launched on EOS Aqua on May 4, 2002 together with ASMU-A and HSB to form a next generation polar orbiting infrared and microwave atmosphere sounding system (Pagano et al 2003). The theoretical approach used to analyze AIRS/AMSU/HSB data in the presence of clouds in the AIRS Science Team Version 3 at-launch algorithm, and that used in the Version 4 post-launch algorithm, have been published previously. Significant theoretical and practical improvements have been made in the analysis of AIRS/AMSU data since the Version 4 algorithm. Most of these have already been incorporated in the AIRS Science Team Version 5 algorithm (Susskind et al 2010), now being used operationally at the Goddard DISC. The AIRS Version 5 retrieval algorithm contains three significant improvements over Version 4. Improved physics in Version 5 allowed for use of AIRS clear column radiances $\widehat{R_{\hat{I}}}$ in the entire $4.3 \mu \mathrm{m} \mathrm{CO}_{2}$ absorption band in the retrieval of temperature profiles $T(p)$ during both day and night. Tropospheric sounding $15 \mu \mathrm{m}$ $\mathrm{CO}_{2}$ observations were used primarily in the generation of clear column radiances $\hat{R}_{\hat{i}}$ for all channels. This new approach allowed for the generation of accurate Quality Controlled values of $\widehat{R_{\hat{I}}}$ and $T(p)$ under more stressing cloud conditions. Secondly, Version 5 contained a new methodology to provide accurate case-bycase error estimates for retrieved geophysical parameters and for channel-by-channel clear column radiances. Thresholds of these error estimates are used in a new approach for Quality Control. Finally, Version 5 contained for the first time an approach to provide AIRS soundings in partially cloudy conditions that does not require use of any microwave data. This new AIRS Only sounding methodology was developed as a backup to AIRS Version 5 should the AMSU-A instrument fail. Susskind et al 2010 shows that Version 5 AIRS Only sounding are only slightly degraded from the AIRS/AMSU soundings, even at large fractional cloud cover.

Significant further progress has been made by the AIRS Science Team since the delivery of the AIRS Version 5 retrieval algorithm to form the AIRS Science Team Version 6 AIRS/AMSU retrieval algorithm. The Version 6 retrieval algorithm is for the most part very similar to Version 5 with one major exception. As in Version 5, the generation of cloud cleared radiances $\hat{R}_{i}$ for all channels is performed using observed radiances $R_{\mathrm{i}}$ for longwave $15 \mu \mathrm{m}$ and $11 \mu \mathrm{m}$ channels. Tropospheric temperature profiles were retrieved in Version 5 
using only the AIRS shortwave $4.2 \mu \mathrm{m} \mathrm{CO}_{2}$ channels, but surface skin temperature $T_{\text {skin }}$ was retrieved simultaneously with surface spectral emissivity and bi-directional reflectance using observations both in the longwave 8-12 $\mu \mathrm{m}$ window region and in the shortwave $4.0 \mu \mathrm{m}-3.76 \mu \mathrm{m}$ window region. In Version 6 , only window observations in the shortwave window region, $4.0 \mu \mathrm{m}-3.76 \mu \mathrm{m}$ are used to determine surface skin temperatures as well as shortwave surface spectral emissivities and surface bi-directional reflectance. The current use of only shortwave AIRS channels in the retrieval of both atmospheric and surface parameters has resulted in significant improvement in the ability to obtain both accurate temperature profiles and surface skin temperatures under more stressing partial cloud cover conditions.

Figure 1 shows a typical AIRS brightness temperature spectrum and includes the channels used in Version 6 for cloud clearing, and in each of the different steps of the AIRS physical retrieval algorithm. Channels used only for cloud clearing are shown in yellow and those used to determine $T(p)$ as shown in red. $\mathrm{CO}_{2}$ channels in the $15 \mu \mathrm{m}$ region sensitive only to stratospheric temperature are used only in the determination of temperature profile and those sensitive to tropospheric temperature are used only for cloud clearing purposes. The channels shown in blue are used both for the determination of $T_{s k i n}$ and $T(p)$.

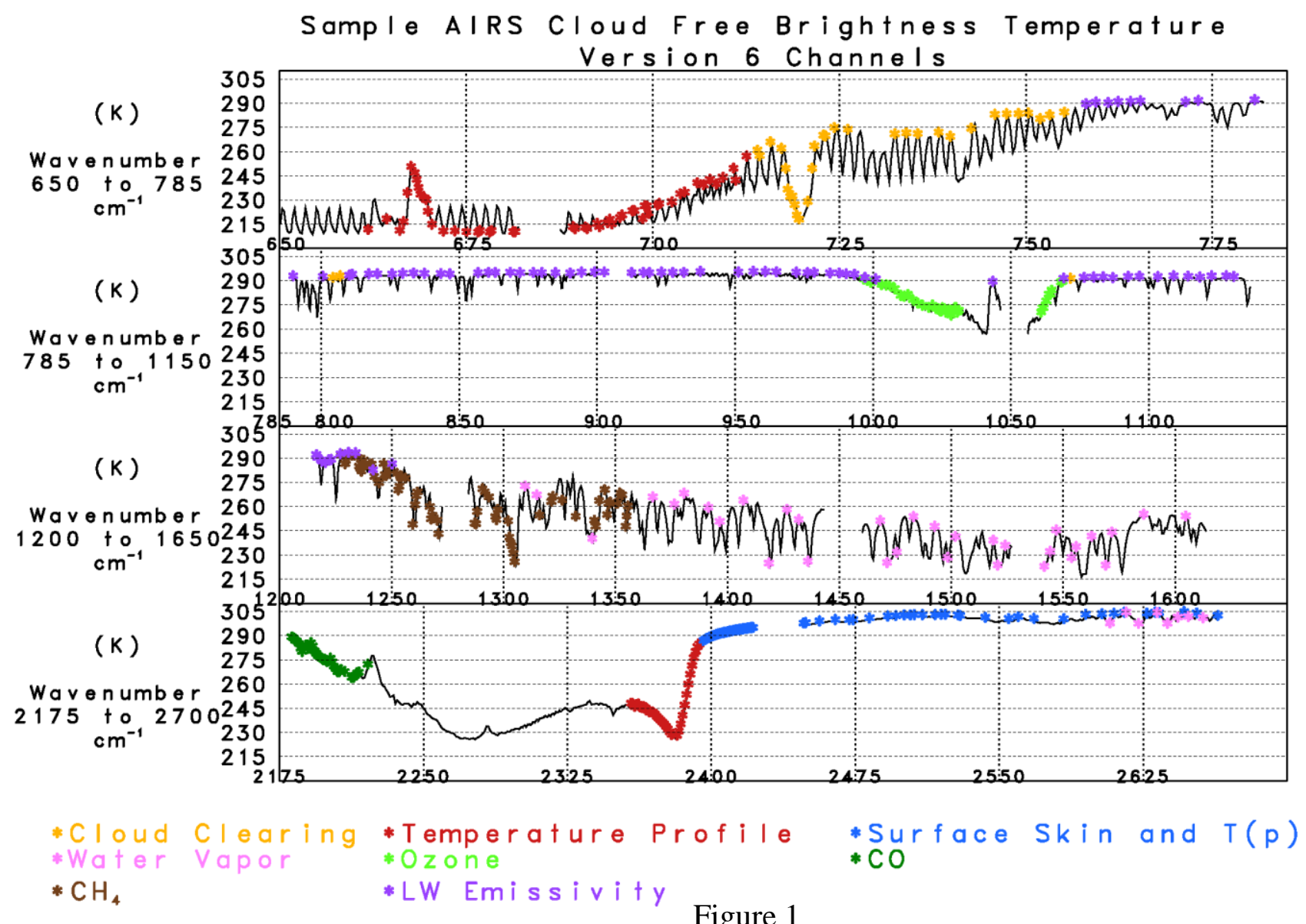

Figure 1 
As with earlier versions, constituent profile retrievals are performed in separate steps, each having their own set of channels and functions. Figure 1 shows in different colors the Version 6 channels used in each of these retrieval steps. The $q(p)$ retrieval (pink stars) uses 33 channels in the spectral ranges $1377 \mathrm{~cm}^{-1}$ to $1605 \mathrm{~cm}^{-1}$ and $2608 \mathrm{~cm}^{-1}$ to $2656 \mathrm{~cm}^{-1}$; the $O_{3}(p)$ retrieval (green stars) uses 41 channels between $997 \mathrm{~cm}^{-1}$ and 1069 $\mathrm{cm}^{-1}$; the $C O(p)$ retrieval (green stars) uses 36 channels between $2181 \mathrm{~cm}^{-1}$ and $2221 \mathrm{~cm}^{-1}$; and the $C_{4}(p)$ retrieval (brown stars) uses 71 channels between $1230 \mathrm{~cm}^{-1}$ and $1356 \mathrm{~cm}^{-1}$.

Version 6 Quality Controlled atmospheric temperature profiles are considerably more accurate than those of version 4, especially under cloudier conditions. The major difference between these two sets of results is due to the fact that most of the tropospheric temperature profile information in Version 4 came from the use of $\widehat{R_{i}}$ for $15 \mu \mathrm{m} \mathrm{CO} \mathrm{CO}_{2}$ channels in the retrieval process, while Version 6 does not use $\widehat{R_{\hat{I}}}$ for any tropospheric sounding $15 \mu \mathrm{m} \mathrm{CO}_{2}$ channels. There were also other differences between Version 4 and Version 6 as well, such as the Quality Control methodology. AIRS Version 5 used both $11 \mu \mathrm{m}$ and $3.7 \mu \mathrm{m}$ window channels in the determination of $T_{\text {skin }}$ over ocean and land areas. The Version 6 algorithm uses only $3.7 \mu \mathrm{m}$ channels for this purpose. In a manner analogous to the methodology in the determination of temperature profiles by using only shortwave $\mathrm{CO}_{2}$ channels for tropospheric temperature sounding, this major change in methodology results in considerable improvement in the ability to obtain accurate SST's under more difficult cloud conditions by using $\widehat{R_{\hat{i}}}$ for only shortwave window regions to solve for $T_{\text {skin }}$ as compared to combined use of $\hat{R}_{\hat{i}}$ in both the longwave and shortwave window regions. Results obtained during the day are at least as good, if not better, than those obtained at night.

This improved sounding methodology is made possible as a result of a few factors, both theoretical and instrumental. First of all, use of improved radiative transfer physics allows for the accurate computation of the effects of non-LTE on the observed radiances in the $4.2 \mu \mathrm{m} \mathrm{CO}_{2}$ band during the day. Secondly, the cloud clearing methodology used to analyze AIRS data removes the effects of solar radiation reflected by clouds from the clear column radiances $\hat{R_{\tilde{i}}}$ used to generate the solution. Effects of solar radiation reflected by the surface on the shortwave radiances are accounted for directly as part of the surface parameter retrieval process. Also, improved error estimates and Quality Control methodology allows for the screening of cases in which aspects of the retrieval methodology may have performed poorly.

Finally, and most significantly, use of these theoretical improvements is made practical because the noise in the AIRS shortwave channels is extremely low. This approach is not practical using IASI data because the IASI noise is too high in the shortwave portion of the spectrum. The findings of this paper are significant with 
regard to design considerations of future high spectral resolution IR sounders, especially for geostationary (GEO) orbit. It is optimal on such sounders to have spectral coverage beyond $2400 \mathrm{~cm}^{-1}$ with very low noise, to produce accurate soundings under most cloud conditions. It is not essential for advanced IR GEO sounder to be accompanied by a GEO microwave sounder to achieve these results.

\section{REFERENCES}

Pagano, T. S., Aumann, H. H., Hagan, D. E. and Overoye, K., "Prelaunch and in-flight radiometric calibration of the Atmospheric Infrared Sounder (AIRS),” IEEE Trans. Geosci. Remote Sensing, 41, 265-273, 2003.

Susskind, J, Blaisdell, J. M., Iredell, L. and Keita, F., "Improved Temperature Sounding and Quality Control Methodology Using AIRS/AMSU Data: The AIRS Science Team Version 5 Retrieval Algorithm, IEEE

Trans. Geosci. Remote Sensing, TGRS-2009-00127-R2, in press, 2010. 


\title{
IMPROVED DETERMINATION OF SURFACE AND ATMOSPHERIC TEMPERATURES USING ONLY SHORTWAVE AIRS CHANNELS: THE AIRS VERSION-6 RETRIEVAL ALGORITHM
}

\author{
Joel Susskind ${ }^{1}$, John Blaisdell ${ }^{2}$, and Lena Iredell ${ }^{2}$ \\ ${ }^{l}$ NASA Goddard Space Flight Center, Greenbelt, MD, USA 20771 \\ ${ }^{2}$ SAIC, NASA Goddard Space Flight Center, Greenbelt, MD, USA 20771
}

ABSTRACT

AIRS was launched on EOS Aqua on May 4, 2002 together with ASMU-A and HSB to form a next generation polar orbiting infrared and microwave atmosphere sounding. The AIRS Science Team Version 6 retrieval system uses only shortwave $\mathrm{CO}_{2}$ channels to determine temperature profile, and only window observations in the shortwave window region, $4.0 \mu m-3.76 \mu m$, to determine both surface skin temperatures and shortwave surface spectral emissivities. The current use of only shortwave AIRS channels in the retrieval of both atmospheric and surface parameters has resulted in significant improvement in the ability to obtain accurate temperature profiles and surface skin temperatures under more stressing partial cloud cover conditions than achieved previously. In this paper, we will show the improvement in retrieved Quality Controlled values of sea surface temperature and ocean spectral surface emissivity compared to those obtained using the AIRS Version-5 retrieval algorithm which used longwave and shortwave window observations simultaneously to determine surface parameters.

\section{INTRODUCTION}

The Goddard DISC uses the AIRS Science Team Version-5 [1] algorithm to analyze AIRS/AMSU data. Improved physics in Version-5 allowed for use of AIRS clear column radiances $\widehat{R}_{i}$ in the entire $4.3 \mu \mathrm{m}$ $\mathrm{CO}_{2}$ absorption band in the retrieval of temperature profiles temperature profile during both day and night. Tropospheric sounding $15 \mu m \mathrm{CO}_{2}$ observations were used primarily in the generation of the clear column radiances $\widehat{R}_{i}$ for all channels. This new approach of determining $\widehat{R}_{i}$ using longwave observations and temperature profile using shortwave observations follows theoretical considerations [2,3], and allows for the generation of accurate Quality Controlled values of $\widehat{R}_{i}$ and temperature profile under more stressing cloud conditions. Version 6 uses not only shortwave channels to determine temperature profile, but also uses only window observations in the shortwave window region, $4.0 \mu m-3.76 \mu m$, to determine surface skin temperatures as well as shortwave surface spectral emissivities and surface bi-directional reflectance. This resulted in significant improvement in the ability to obtain accurate surface skin temperatures as well as temperature profiles under more stressing partial cloud cover conditions.

Index Terms - Infra-red measurements, remote sensing, meteorology, sea surface

\section{CHANNELS USED IN THE VERSION-6 PROCESSING SYSTEM}

Figure 1 shows a typical AIRS brightness temperature spectrum and includes the channels used in Version-6 for cloud clearing, and in each of the different steps of the AIRS physical retrieval algorithm. Channels used only for cloud clearing are shown in yellow and those used to determine temperature profile are shown in red. $\mathrm{CO}_{2}$ channels in the $15 \mu \mathrm{m}$ region sensitive only to stratospheric temperature are used only in the determination of temperature profile and those sensitive to tropospheric temperature are used only for cloud clearing purposes. The channels shown in blue are used both for the determination of $T_{\text {skin }}$ and temperature profile. Longwave window channels shown in purple are used for the determination of the longwave surface spectral emissivity. 


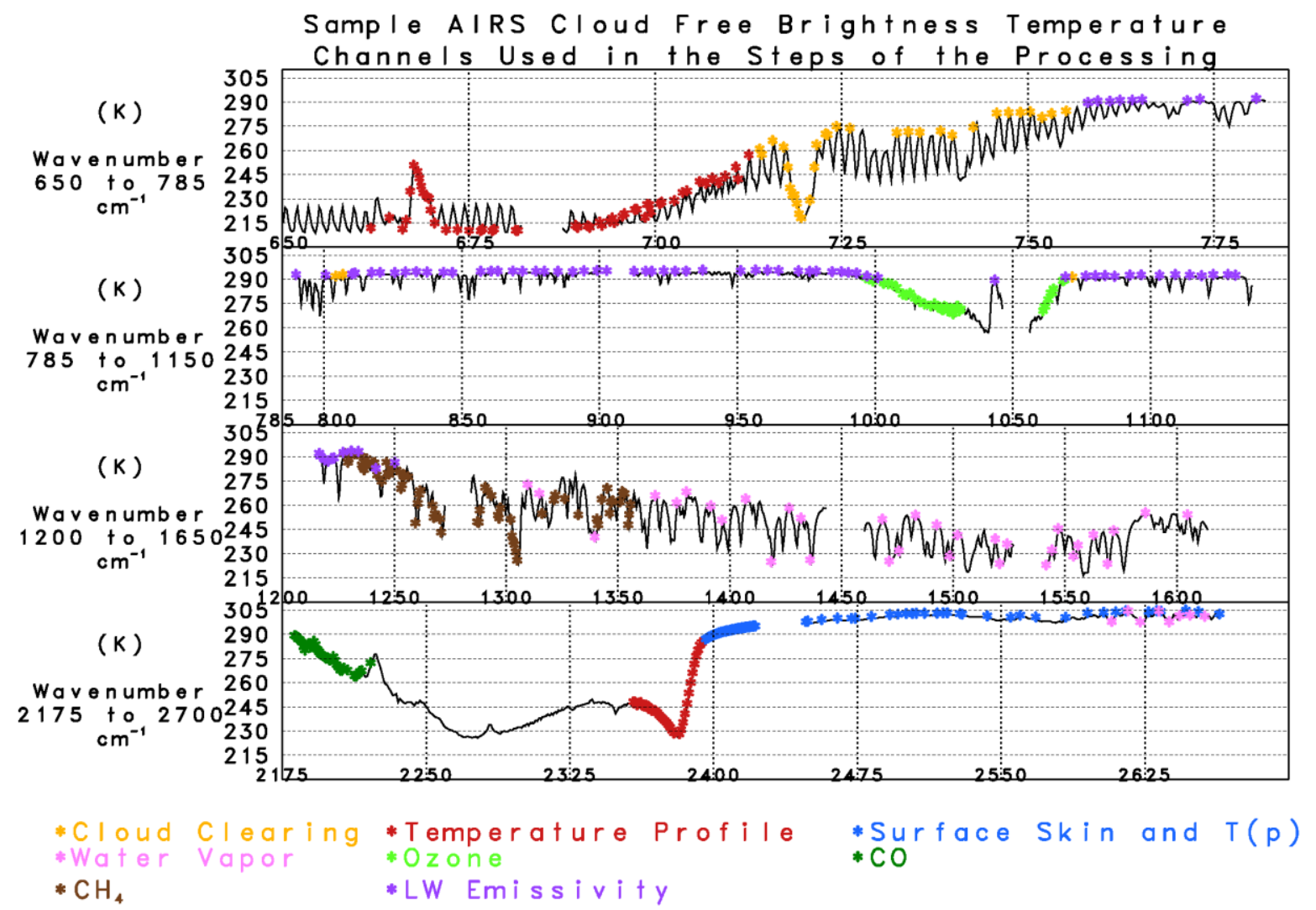

Figure 1

As with earlier versions, constituent profile retrievals are performed in separate steps, each having their own set of channels and functions. The water profile retrieval (pink stars) uses 33 channels in the spectral ranges $1377 \mathrm{~cm}^{-1}$ to $1605 \mathrm{~cm}^{-1}$ and $2608 \mathrm{~cm}^{-1}$ to $2656 \mathrm{~cm}^{-1}$; the ozone profile retrieval (green stars) uses 41 channels between $997 \mathrm{~cm}^{-1}$ and $1069 \mathrm{~cm}^{-1}$; the $\mathrm{CO}$ profile retrieval (green stars) uses 36 channels between $2181 \mathrm{~cm}^{-1}$ and $2221 \mathrm{~cm}^{-1}$; and the $\mathrm{CH}_{4}$ profile retrieval (brown stars) uses 71 channels between 1230 $\mathrm{cm}^{-1}$ and $1356 \mathrm{~cm}^{-1}$. These same channels are used in the Version-6AO "AIRS Only" retrieval system that does not include use of any AMSU channels.

\section{IMPROVEMENTS IN SEA SURFACE TEMPERATURE}

Figure 2 shows histograms of the counts of errors of Quality Controlled ocean sea surface temperature (SST) retrievals as a function of SST differences from ECMWF "truth" for an average of 6 days ranging from September 6, 2002, through August 10, 2007. Results are shown for Quality Controlled SST retrievals observed using Version-5, Version-6, and Version$6 \mathrm{AO}$. The mean difference from ECMWF, the spatial standard deviation of ECMWF, the spatial standard deviation of the difference, the percent of all cases accepted, and the percent outliers (errors more than $3 \mathrm{~K}$ from the mean) are indicated. Results for day and night are shown separately. Version-6 Quality Controlled SST retrievals have considerably higher yields with similar standard deviations and percent outliers than the comparable Quality Controlled Version-5 SST's. The accuracy of the Quality Controlled retrievals, as well as the improvement over Version-5, is actually greater during the day than at night even though the shortwave window channel observations are affected significantly by solar radiation reflected by clouds and the surface. It is noteworthy that the quality of the AIRS Only retrieval system Version-6AO is only slightly degraded from that of Version-6. The same has been found for atmospheric temperature profile [1]. This implies that it is not necessary to have a microwave sounder to accompany an AIRS-like instrument to perform accurate soundings under partial cloud cover.

The Quality Controlled AIRS SST's are used to generate Level-3 monthly mean products on a $1^{\circ} \mathrm{x} 1^{\circ}$ lat-lon grid for the purpose of studying climate variability and trends. For these purposes, it is 

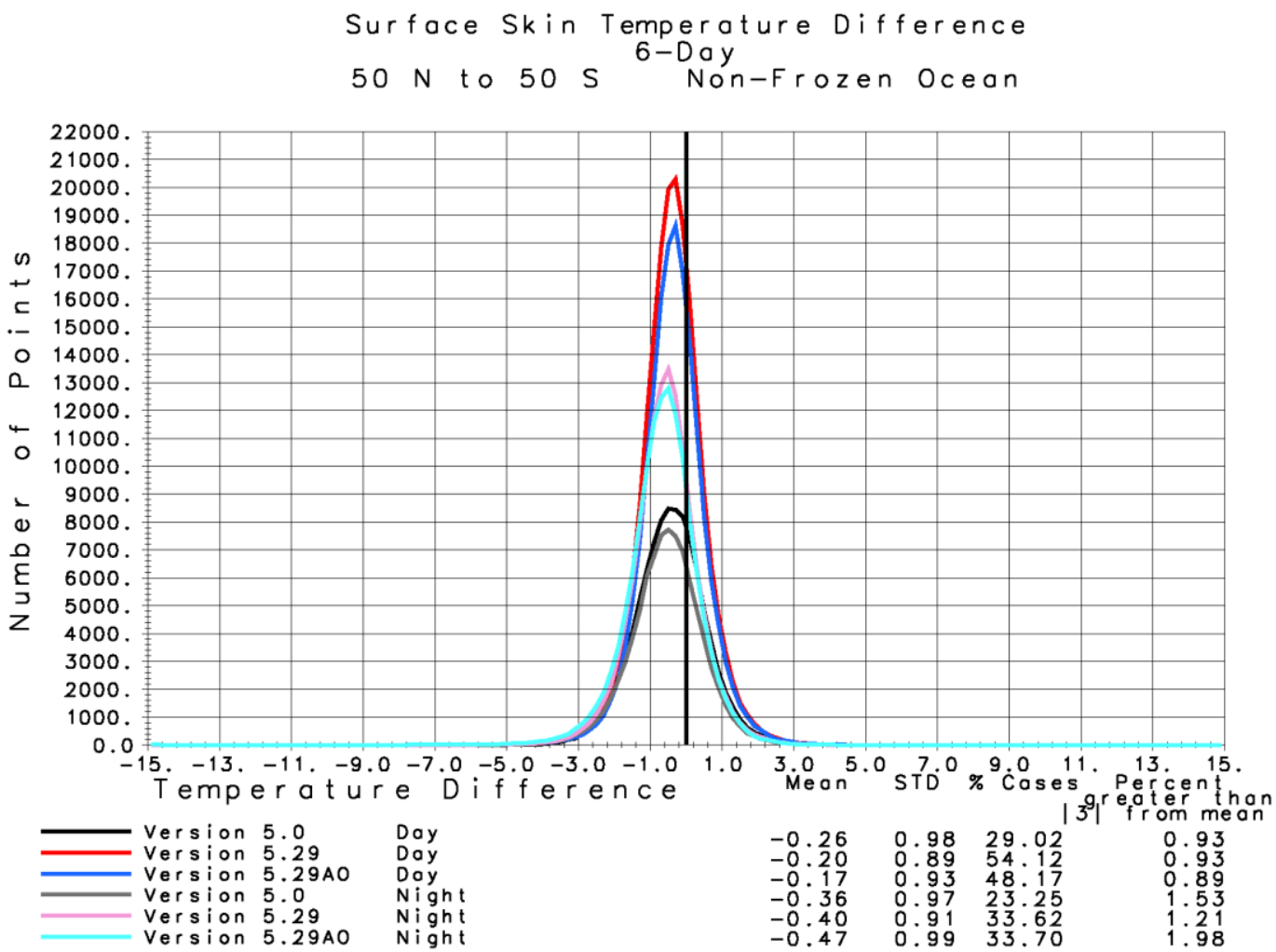

Figure 2

Surface Skin Temperature Version-6

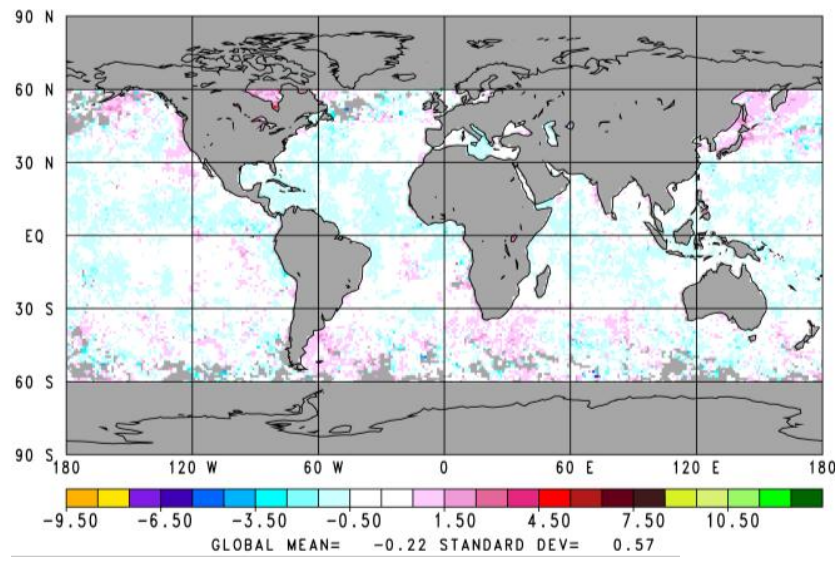

Retrieved minus ECMWF

\section{Version-5}

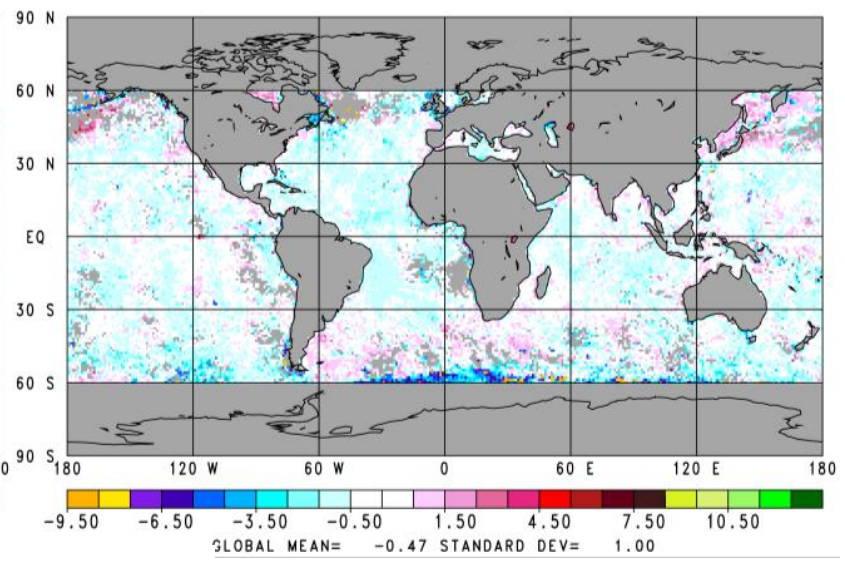

Figure 3

important to have both good spatial coverage and accuracy. Figure 3 shows the spatial distribution of 6day mean Version-6 and Version-5 Quality Controlled SST's, $60^{\circ} \mathrm{N}-60^{\circ} \mathrm{S}$, as well as their average difference from ECMWF "Truth". Both 1:30 AM and 1:30 PM observations have been included in the generation of Figure 3. The spatial coverage of Quality Controlled 6day mean SST fields, as well as their accuracy, are much better in Version-6 than in Version-5. Note in particular the better coverage and high accuracy of Version-6 SST's in perpetually cloudy regions off the west coast of South America and Africa. This is a direct result of the ability to generate improved Quality Controlled SST soundings under more stressing cloud conditions by using only shortwave channels to determine SST. 
Figures 4a and 4b show results using Quality Controlled retrieved ocean surface spectral emissivity obtained using Version-5, Version-6, and Version6AO. Surface spectral emissivity over ocean depends to first order on frequency, wind speed, and the zenith angle of observation. In principle, there should be little difference of ocean surface spectral emissivity between day and night. The degree, if any, of day minus night retrieved surface spectral emissivity is a good test of how well the retrieval algorithms account for the effects of solar radiation, reflected both by clouds and the surface, on the observed radiances. The mean value of spectral emissivity retrieved during the day minus that retrieved at night is shown as a function of observed satellite zenith angle. A negative zenith angle indicates scanning to the left of nadir viewing and a positive zenith angle indicates scanning to the right. At night, reflected solar radiation does not affect radiances at any zenith angle. During the day, solar radiation reflected by the ocean surface has the largest effect at a satellite zenith angle near $-18^{\circ}$ because the ocean provides the largest sunglint at this viewing angle.

Figure $4 \mathrm{a}$ shows results for ocean surface emissivity at $2500 \mathrm{~cm}^{-1}$, a window frequency very sensitive to reflected solar radiation. The day/night difference of spectral emissivity is relatively small (though not zero) at all angles for both Version-6 and Version-6AO. This indicates that the surface parameter retrieval algorithm, which uses only shortwave window channels to simultaneously determine skin temperature, surface shortwave spectral emissivity, and surface spectral bi-directional reflectance, is performing very well during the day, even in regions of sunglint. The Version-5 surface spectral emissivity at $2500 \mathrm{~cm}^{-1}$, retrieved using both longwave window and shortwave window observations simultaneously, is of much poorer quality than those in Version-6 and 6AO, especially in the presence of sunglint. Figure $4 \mathrm{~b}$ shows analogous plots for surface spectral emissivity at $850 \mathrm{~cm}^{-1}$ in the longwave window region. As with Figure 4a, Version5 retrieved spectral emissivity at $850 \mathrm{~cm}^{-1}$, in a spectral region unaffected by reflected solar radiation, performed poorly during the day especially in sunglint areas.

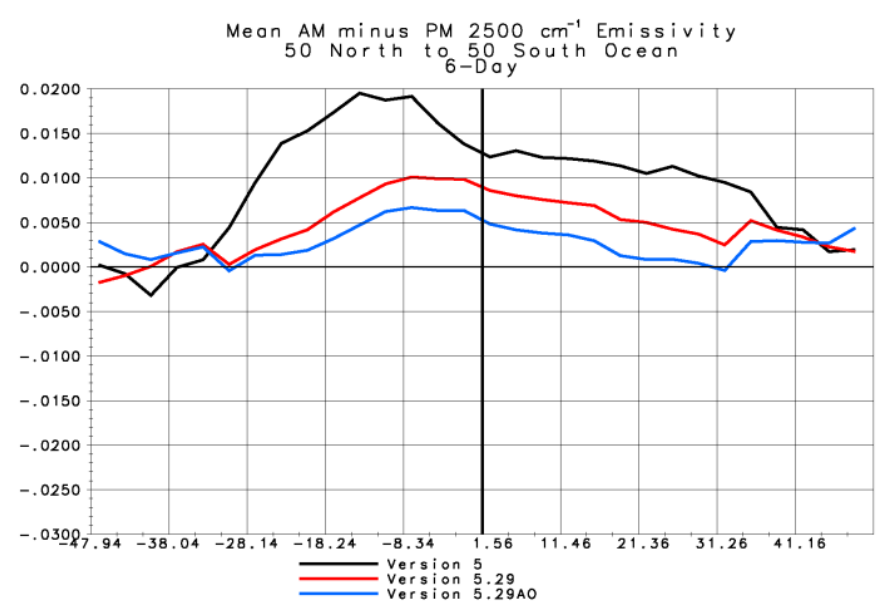

Figure $4 \mathrm{a}$

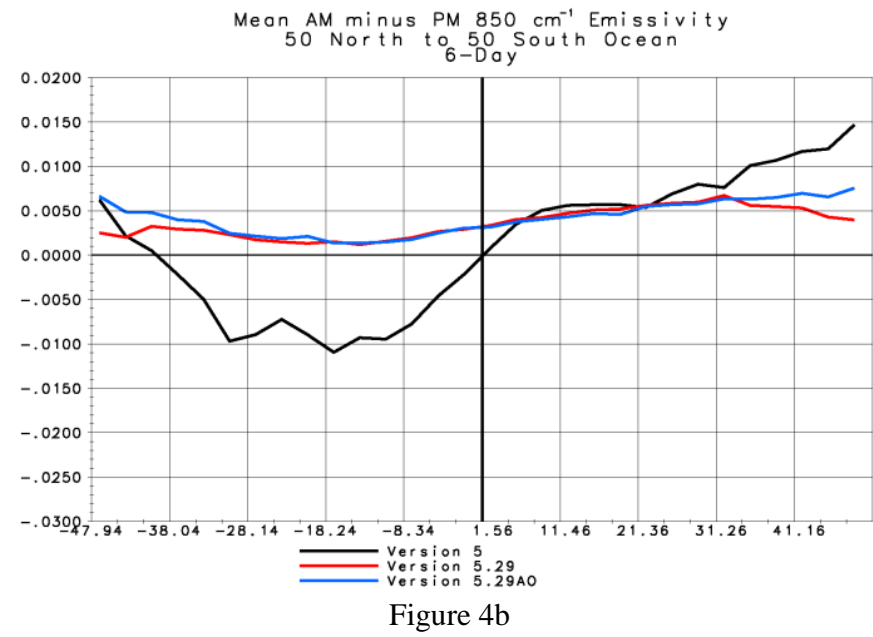

\section{REFERENCES}

[1] M. T. Chahine, "Remote Sensing of Cloudy Atmospheres. I. The Single Cloud Layer," J. Atmos. Sci., vol. 31, pp. 233-243, 1974.

[2] M. T. Chahine, "Remote Sensing of Cloudy Atmospheres. II. Multiple Cloud Formations," J. Atmos. Sci., vol. 34, pp. 744-757, 1977.

[3] J. Susskind, J. M. Blaisdell, L. Iredell, and F. Keita, "Improved Temperature Sounding and Quality Control Methodology Using AIRS/AMSU Data: The AIRS Science Team Version-5 Retrieval Algorithm." In press, IEEE Trans. On Geoscience and Remote Sensing, 2010. 